\title{
EL COSTO DE LOS NIÑOSY SU IMPACTO EN EL PRESUPUESTO DE LAS FAMILIAS. UN ANALISIS PARA ARGENTINA
}

THE COST OF CHILDREN AND ITS IMPACT ON THE FAMILY BUDGET. AN APPLICATION TO ARGENTINA

\section{MIRIAM BERGES* \\ Universidad Nacional de Mar del Plata}

\section{LUCIA ECHEVERRIA**}

CONICET - Universidad Nacional de Mar del Plata

\section{JUAN IGNACIO RODRIGUEZ BIASONE***}

Universidad Nacional de Mar del Plata

\begin{abstract}
Resumen
Las escalas de equivalencia son fundamentales para realizar análisis de bienestar. Sin embargo, la evidencia para América Latina es escasa. Este trabajo estima el costo de los niños para familias biparentales y monoparentales en Argentina a partir de datos de gasto. El enfoque se basa en el modelo de Rothbarth, en donde el bienestar de los padres depende del gasto en bienes de adultos. El costo de los niños es creciente con el número de niños, pero existen economías de escala. Asimismo, las escalas difieren según la estructura familiar, y del sexo y composición etaria de los niños.
\end{abstract}

Palabras claves: Escalas de equivalencia, método de Rothbarth, bienestar, costo de los niños.

Clasificación JEL: D12, I31.

* Facultad de Ciencias Económicas y Sociales, Universidad Nacional de Mar del Plata. Argentina. E-mail: mberges@mdp.edu.ar

** CONICET (Consejo Nacional de Investigaciones Científicas y Técnicas). Argentina. E-mail: lecheverria@ mdp.edu.ar

*** Facultad de Ciencias Económicas y Sociales, Universidad Nacional de Mar del Plata. Argentina. E-mail: rodbjuanignacio@gmail.com 


\begin{abstract}
Equivalence scales serve a key role in welfare analysis. However, the empirical evidence for Latin American countries has been relatively scarce. We estimate the cost of children for two-parent and single-parent families in Argentina using consumption data. The methodology follows the Rothbarth approach, in which the welfare of the parents depends on the expenditure in adult goods. Results indicate that the cost of children is increasing in the number of children in the household, but economies of scales at the family level are observed. Further, scales differ according to the family structure, and sex and age composition of the children.
\end{abstract}

Keywords: Equivalence scales, Rothbarth approach, welfare, cost of children.

JEL Classification: D12, I31.

\title{
1. INTRODUCCION
}

El concepto de bienestar involucra tanto aspectos objetivos como subjetivos de las personas, en tanto aproximan su calidad de vida. Sin embargo, debido a la dificultad de observar y capturar estos últimos, el nivel de bienestar suele ser aproximado utilizando alguna dimensión monetaria. Desde esta perspectiva, los hacedores de política que buscan mejorar el bienestar de la población suelen centrarse en el ingreso como uno de los ejes clave para realizar diagnósticos y diseñar programas.

Las mediciones de bienestar suelen enfocarse en la distribución personal de los ingresos, sin embargo la información de microdatos es relevada frecuentemente a nivel de los hogares. El ingreso de los hogares no constituye una medida adecuada del bienestar, en tanto no considera la estructura demográfica de las familias. Por tanto, es deseable emplear algún indicador que permita ajustar el ingreso total del hogar por su composición demográfica para realizar evaluaciones de bienestar. Las escalas de equivalencia cumplen este rol, al permitir comparar los ingresos de hogares con distinta composición demográfica tomando como referencia un tipo de hogar determinado, compuesto generalmente por un hombre adulto (Lazear y Michael, 1980; Deaton, 1997).

En la literatura internacional existe una amplia variedad de escalas de equivalencia, sustentadas en distintos enfoques y supuestos de identificación. Sin embargo, no existe consenso acerca de cuál resulta la más adecuada para realizar análisis de bienestar, tales como las mediciones de pobreza o desigualdad. En este sentido, la evidencia sugiere que la elección del ajuste demográfico elegido en estudios distributivos impacta notoriamente los niveles de pobreza y la composición de la población identificada como pobre (Coulter, Cowell y Jenkins, 1992; De Vos y Zaidi, 1997; Betson y Muellbauer, 2004). 
Pese a la larga tradición que han tenido las escalas de equivalencia en la literatura internacional, su tratamiento empírico ha sido relativamente escaso en los países de América Latina (Alonzo y Mancero, 2011). En Argentina no se encuentran antecedentes empíricos de estimación de escalas de equivalencia hasta los trabajos de Berges (2010, 2011), quien calcula escalas IB, estimadas bajo el supuesto de independencia del nivel de ingreso base de referencia (Blackorby y Donaldson, 1988; Lewbel, 1989). Las escalas obtenidas a nivel país con encuestas de gasto para el período 1996/97 presentan evidencia de importantes economías de escala en el consumo de los hogares a medida que aumenta el número de integrantes. Sin embargo, las escalas más altas que se obtienen con datos de 2004/05 permiten observar una disminución en dichas economías de escala así como un aumento en la fracción que representan los gastos de un niño respecto de los de un adulto. Adicionalmente, Echeverría y Berges (2015) estiman escalas asociadas a un nivel de subsistencia para hogares de la Ciudad Autónoma de Buenos Aires, mientras que Pace Guerrero (2013) estima escalas de Barten por tipo de integrantes y por tipo de gasto para todos los hogares del país.

Este trabajo se focaliza en analizar el costo adicional que impone la incorporación de los niños al hogar bajo un enfoque alternativo y toma en consideración dos tipos de hogares nucleares: monoparentales y biparentales. En particular, se estiman escalas de equivalencia bajo el método de Rothbarth para hogares con distinto número de niños. Adicionalmente, se propone el cómputo de escalas considerando el grupo etario y el sexo de los niños. La estrategia empírica está basada en Schulte (2007), aunque este autor no realiza corrección alguna del potencial sesgo de selección presente en los datos de consumo ${ }^{1}$. Los datos utilizados son los provenientes de la Encuesta Nacional de Gasto de los Hogares 2012/13 de Argentina, elaborada por el Instituto Nacional de Estadística y Censos (INDEC).

El enfoque adoptado permite capturar el costo de un niño a partir de los efectos de ingreso que se generan en el consumo de los denominados "bienes de adultos", esto es, aquellos bienes que son consumidos exclusivamente por los miembros adultos del hogar (como, por ejemplo, la ropa de adultos). Las escalas de Rothbarth están basadas en el supuesto de que el bienestar de los adultos está directamente vinculado al nivel de gasto en los bienes adultos, tal que los adultos de dos hogares distintos tendrán el mismo nivel de bienestar si gastan la misma cantidad de dinero en ese tipo de bienes. De esta forma, las escalas indican el costo en el que incurre una familia con hijos para que los adultos alcancen el mismo nivel de bienestar que tendrían en caso de no tener hijos.

Las escalas de equivalencia estimadas indican que el costo de los niños es creciente a medida que se incorporan más niños al hogar. Sin embargo, se observa evidencia de economías de escala en el consumo de la familia en tanto los incrementos son

1 Según consigna el autor en el trabajo citado, sus datos presentan una proporción insignificante de observaciones cero. 
proporcionalmente menores. La evidencia indica que el costo de los niños es mayor para los hogares monoparentales. Asimismo, las escalas de equivalencia correspondientes a una misma cantidad de niños en el hogar difieren en función del sexo y la edad de los niños. Si los niños en un mismo hogar pertenecen a rangos etarios diferentes -según sean menores o mayores de cinco años-, los costos que enfrenta el hogar para mantener el bienestar de referencia serán más elevados. A su vez, mientras que las niñas son relativamente más caras para el hogar de una pareja, los varones lo son para el hogar monoparental.

El trabajo contribuye a la literatura empírica de las escalas de equivalencia con evidencia reciente de escalas estimadas por el método de Rothbarth para Argentina. Asimismo, la diferenciación entre hogares compuestos por dos padres o por solo uno de ellos no es trivial para el diseño de políticas, porque una alta proporción de hogares a cargo de un único miembro son hogares bajo la línea de pobreza. Además, este trabajo es el primero en estimar escalas de Rothbarth diferenciando la edad y el género de los niños. Los resultados de este trabajo constituyen mediciones alternativas a las utilizadas en el cómputo de la pobreza que realiza actualmente el INDEC y que están basadas en los requerimientos calóricos que corresponden a los miembros del hogar. También permiten evaluar y discutir las diferencias existentes con otras escalas estimadas bajo distintos métodos y supuestos de identificación. Por último, a partir de estos resultados es posible generar recomendaciones que podrían ser de utilidad para discutir las políticas de transferencias focalizadas en las familias con niños.

El trabajo está estructurado de la siguiente manera. En la Sección 2 se explica el marco teórico referido a las escalas de equivalencia y el método de Rothbarth. En la Sección 3 se detallan los datos empleados y se aportan estadísticas descriptivas como análisis preliminar. En la Sección 4 se describe en detalle la estrategia empírica adoptada. En la Sección 5 se presentan los resultados de las escalas de equivalencia estimadas, mientras que en la Sección 6 se presentan las conclusiones y recomendaciones de política.

\section{ESCALAS DE EQUIVALENCIA}

Las escalas de equivalencia son indicadores que permiten expresar el ingreso nominal de hogares con distinta composición demográfica en términos de unidades homogéneas, lo que significa que determinado ingreso nominal en un hogar es equivalente a " $\mathrm{X}$ " unidades del ingreso de otro hogar con distintas características demográficas (Lazear y Michael, 1980). Es decir, las escalas de equivalencia se definen como un índice que muestra, a precios de referencia, la diferencia de costos en la que debe incurrir un cierto hogar, debido a su tamaño y composición, para alcanzar la curva de indiferencia del hogar de referencia (Grootaert, 1982).

Existe una amplia variedad de escalas de equivalencia, construidas bajo distintos métodos y supuestos. Entre ellas, las estimadas a base de valores estipulados por 
expertos (comúnmente requerimientos de calorías de acuerdo con edad y género de los miembros), las que se basan en parámetros fijos para considerar economías de escala y el peso relativo de cada tipo de miembro en el hogar, las "subjetivas" que se obtienen a partir de encuestas directas a los hogares y las que se basan en el comportamiento de consumo de los hogares. Este último enfoque es el que propone la teoría económica, empleando encuestas de gastos a los hogares con las cuales es posible observar los patrones de consumo revelados por las familias por medio de sus gastos. Estas escalas se obtienen a partir de estimaciones realizadas con los datos reales de lo que efectivamente gastan los hogares, en función de su composición demográfica (Deaton y Muellbauer, 1980). Las escalas de Rothbarth se encuentran dentro de este último grupo.

Para medir los niveles de bienestar de distintos tipos de hogares se asume que la función de utilidad directa de los padres depende de las mercancías consumidas por el hogar y las características demográficas del mismo. Asociada a ella, hay una función de costo que proporciona el nivel mínimo de gasto, $X$, necesario para alcanzar el nivel de utilidad $U$, a precios $p$, con las características demográficas del hogar $z$ (Tsakloglou, 1991). Entonces, se puede utilizar dicha función para calcular el costo adicional en que debe incurrir un hogar determinado para alcanzar el nivel de bienestar del hogar de referencia.

Sea $C=C(U, p, k) C$ el costo que tiene un determinado hogar para alcanzar un nivel de bienestar $U$ dado el vector de precios $p$ y la composición del hogar $k$, las escalas de equivalencia se definen de acuerdo con la expresión (1).

$$
m=\frac{C(U, p, k)}{C\left(U, p, k_{0}\right)}
$$

De esta manera, las escalas definen el costo relativo de alcanzar un nivel de bienestar $U$, dado un vector de precios $p$, para un hogar de composición $k$, en comparación con un hogar de características $k_{0}$. Si $k_{0}$ denota una pareja sin hijos y $k$ una pareja con un niño, utilizando el nivel el nivel de utilidad antes del niño, a los mismos precios base, el exceso de la ecuación (1) con respecto a uno representa el costo de un niño como proporción de los gastos del hogar. La diferencia en los costos del hogar representa el monto de dinero necesario para restablecer el nivel de utilidad original, dada la nueva composición del hogar. Sin embargo, este procedimiento requiere de información adicional que permita la identificación de las escalas.

Los problemas de identificación podrían agruparse principalmente en torno a dos cuestiones: la naturaleza ordinal de la función de utilidad, y los alcances y limitaciones de la definición de bienestar o utilidad del un hogar. La primera se relaciona con el hecho de que las observaciones de gastos de los hogares son consistentes con más de una función de utilidad, de la que podrían provenir, y es necesario explicitar algún criterio con el que se pueda definir que los hogares obtienen un mismo nivel de 
utilidad. La segunda se relaciona con la dificultad para definir el concepto "utilidad del hogar", cuando son los individuos que lo componen quienen perciben utilidad. Distintos hogares podrían revelar idénticos comportamientos de consumo, sin embargo, al interior de cada uno de ellos los bienes podrían asignarse de manera diferente entre sus miembros y afectar los respectivos niveles de bienestar. También es cierto que la utilidad del hogar podría incluir aspectos subjetivos, así como los derivados de una cierta composición familiar, tener hijos o no, vivir solo o en pareja, pero ese componente de la utilidad no podría verse reflejado en sus gastos de consumo. Entonces, los únicos aspectos que se incluyen son los referidos al bienestar material de los individuos que componen el hogar.

El análisis de las escalas de equivalencia requiere asumir que las funciones de utilidad de un hogar son comparables a las de un individuo, en tanto indican cuánto más ingreso necesita gastar una familia de determinada composición para tener el mismo nivel de bienestar que una persona viviendo sola. Esta limitación puede superarse estimando una versión de las escalas de equivalencia basadas en el bienestar individual, denominadas escalas de indiferencia (Lewbel, 2003; Browning, Chiappori y Lewbel, 2013; Chiappori, 2016) en el marco de los modelos colectivos (Chiappori, 1988, 1992). Estas escalas indican cuánto más ingreso requiere un individuo que vive solo para alcanzar la misma curva de indiferencia que obtendría si fuera miembro de un hogar de determinada composición. Sin embargo, la evidencia de estimación de escalas de indiferencia es muy escasa y reciente (Bargain y Donni, 2012; Bargain, Donni y Kwenda, 2014).

Otra cuestión, relacionada con la información necesaria para determinar las escalas, es la que discute acerca de considerar la totalidad del gasto de las familias o solo un subconjunto de ellos. En muchos de los casos, la presencia de un niño adicional en el hogar no incrementa el presupuesto, sino que reasigna los mismos gastos de otra forma. Y, al no haber modificación del gasto total, no podría determinarse cuánto más gasta una familia debido al cambio en su composición, ni tampoco podrían calcularse las escalas empleando esa variable en las estimaciones. Si se decide, en cambio, emplear solo un subconjunto de bienes, el hecho de incluir o no algunos de ellos, afectaría el valor de las escalas.

Finalmente, estos argumentos no implican la imposibilidad de estimar escalas con base en la teoría del consumo, sino que refuerzan la necesidad de explicitar claramente los supuestos a partir de los cuales han sido obtenidas. Por lo general, toda investigación empírica respecto de consumo y bienestar de los hogares implica algún juicio normativo, implícito en los supuestos que se establecen. Los modelos que se han empleado para estimar escalas de este tipo se basan en la incorporación de una distribución del consumo intrahogar, como los de Rothbarth (en el que se basa este trabajo), Barten o los modelos de negociación colectiva, o establecen algún criterio para definir las condiciones mediante las cuales el bienestar de un hogar es equivalente, como Engel. También es posible emplear supuestos acerca de la forma funcional de las escalas, compatibles con cierto tipo de funciones de utilidad, como las escalas IB (independientes de la base o utilidad de referencia). 


\subsection{Escalas de Rothbarth}

El método de Rothbarth (1943) es uno de los abordajes presentes en la literatura, que parte del supuesto que el bienestar de los niños es una función creciente del bienestar de los padres. Bajo este enfoque, el indicador de bienestar de los padres está dado por el nivel de consumo de los denominados "bienes de adultos", esto es, aquellos bienes que son demandados exclusivamente por los miembros adultos del hogar. De esta manera, los adultos de dos hogares distintos tendrán el mismo nivel de bienestar si gastan la misma cantidad de dinero en bienes de adultos. La particularidad de estos bienes es que deben ser consumidos exclusivamente por adultos, y su consumo no debe verse afectado por la presencia de niños en el hogar ${ }^{2}$. Además, la elasticidad de ingreso de estos bienes debe ser positiva y no cercana a 0 , de forma tal que cambios en las características demográficas del hogar provoquen efectos de ingreso en la demanda de los bienes de adulto (Deaton, 1997).

El método captura efectos ingreso de reasignación, partiendo de la hipótesis que, dado un cierto nivel de ingreso, los hogares con hijos gastan menos en bienes de adulto que aquellos que no los tienen. Entonces, para alcanzar el mismo nivel de bienestar (esto es, el mismo gasto en bienes de adulto), el hogar con niños requerirá un ingreso mayor. Si para tener un gasto determinado en bienes de adulto, el hogar de referencia requiere un ingreso $Y_{0}$ y el hogar con un niño necesita $Y_{1}$, se concluye que el costo de un niño es $Y_{1}-Y_{0}$, mientras que la escala de equivalencia de ese hogar es $Y_{1} / Y_{0}$. El costo de un niño indica, entonces, cuál es la compensación monetaria requerida por el hogar con un niño, para tener el mismo nivel de bienestar que tenía previo a la llegada del niño.

Bajo este enfoque las escalas que se obtienen son solo aplicables a los niños, debido a que resultaría poco plausible argumentar que otros miembros adultos no consumieran los bienes de adulto seleccionados para las estimaciones. Esta es una primera limitación empírica del alcance de este modelo que explica por qué su uso se encuentra siempre asociado al costo de un niño adicional en el hogar. También el modelo enfrenta otras limitaciones, de carácter teórico, que han dado lugar a una extensa literatura en el tema de escalas de equivalencia junto con nuevos enfoques, tampoco exentos de supuestos y nuevas críticas. En particular, el modelo de Rothbarth supone que la utilidad de los padres depende exclusivamente del presupuesto asignado a su propio consumo y es independiente del consumo de los niños. Este supuesto puede rebatirse argumentando que muchos padres son felices en la medida que mejora el bienestar de sus hijos y su utilidad, entonces, aumentaría con el consumo de los niños. Este argumento, sin embargo, no invalida el uso del modelo. En todo caso advierte que "las verdaderas escalas" deberían ser más elevadas, porque el modelo subestimaría

2 Debido a la presencia de niños, los padres podrían elegir, por ejemplo, una menor cantidad de bienes que no permitieran ser disfrutados en familia, así como cierto tipo de espectáculos. 
el costo de los niños para los padres, que deberían obtener mayores compensaciones para alcanzar el nivel de bienestar original ${ }^{3}$.

El modelo de Rothbarth identifica una distribución de recursos en el hogar asumiendo que hay bienes solo consumidos por cierto grupo de miembros. Implícitas en este supuesto, existen al menos dos condiciones que deben cumplirse. En primer lugar, los adultos deben tener preferencias estables, es decir, las preferencias de los padres de su propio consumo no deben modificarse ante la presencia de niños, tema relacionado con lo comentado en el párrafo anterior. En segundo lugar, se debe satisfacer la separabilidad demográfica (Deaton, Ruiz-Castillo y Thomas, 1989), lo que significa que el consumo de bienes de adulto solo puede verse afectado por la presencia de niños mediante el efecto ingreso. Este comportamiento supone la ausencia de cambios en el costo relativo de los bienes frente a la llegada de los hijos y posibles sustituciones en contra de aquellos bienes que podrían ser consumidos tanto por adultos como por los niños ${ }^{4}$. Bajo preferencias estables y separabilidad demográfica se sostiene que la incorporación de un niño al hogar reduce el consumo de bienes de adulto debido al menor presupuesto disponible para gastar en ellos.

La condición de separabilidad también podría verse afectada por la existencia de bienes públicos en el hogar -como la vivienda, la energía y la calefacción, entre otros- asociados con grandes economías de escala en su consumo. Sin embargo, no está demostrado empíricamente en qué medida este aspecto modificaría los resultados obtenidos bajo los supuestos de Rothbarth (Mancero, 2001).

Adicionalmente, existe otra limitación de orden empírico en el caso de algunos bienes de adulto, como alcohol y tabaco. Si su demanda fuera relativamente inelástica respecto del gasto total, no sería posible captar apropiadamente el efecto ingreso. En ese caso, de acuerdo con Tsakloglou (1991) las escalas de Rothbarth tienden a subestimar el costo de un niño, aunque Deaton (1997) indica que en la mayoría de los países en los que ha realizado sus estudios, los gastos en bienes de adulto, incluyendo alcohol y tabaco, responden fuertemente a los cambios en el presupuesto.

Siguiendo a Nelson (1992), el problema al que se enfrentan los hogares con niños es la maximización de la función de utilidad $U\left[u^{a}\left(q^{a}\right), u^{c}\left(q^{c}\right)\right]$, tal que $u^{a}\left(q^{a}\right)$ es la subfunción de utilidad de los adultos definida sobre su propio consumo de bienes $q$, y $u^{c}\left(q^{c}\right)$ es una subfunción de utilidad que se define sobre el consumo de los niños. Los bienes colectivos son ignorados. De acuerdo con el supuesto de preferencias estables, la subfunción $u^{a}\left(q^{a}\right)$ no depende de la presencia de niños en el hogar, ya que los adultos tienen las mismas preferencias sobre su consumo. A su vez, el supuesto de separabilidad implica que la función de demanda de cualquier bien de adulto es $q_{i}{ }^{a}=$

3 "Tal como lo explica Gronau, si los padres derivan utilidad del consumo realizado por sus hijos, la propensión marginal a gastar en 'bienes de adulto’ se verá reducida ante la presencia de hijos adicionales. Si esto ocurre, el método de Rothbarth subestima el costo de un niño...” (Mancero, 2001, pág. 14).

4 Por ejemplo, un padre que hubiera consumido una gaseosa estando solo, podría cambiar su decisión en presencia de su hijo para no enfrentar un costo que fuera el doble de lo previsto (Deaton, 1997). 
$g_{i}{ }^{a}\left(p, x^{a}\right)$, siendo $x^{a}$ el ingreso familiar asignado a los bienes de adulto y $p$ el vector de precios de mercado de los bienes $q$. Si se cumplen las condiciones mencionadas, la llegada de un niño al hogar solo afecta al consumo de bienes de adultos mediante cambios en $x^{a}$. Respecto de la misma curva de indiferencia, la tasa marginal de sustitución entre bienes no debería cambiar con la presencia de niños.

Deaton, Ruiz-Castillo y Thomas (1989) desarrollan un modelo de separabilidad de costos, en el que la función de costos del hogar se divide en dos partes, la primera parte contiene las características demográficas que no están asociadas con los niños y los precios de los bienes de adultos, mientras que la segunda parte contiene los precios de todos los bienes a excepción de los de los bienes de adultos, y las características de los niños. Para llevar a cabo el análisis, es necesario definir un criterio de asignación del gasto en dos etapas. Primero, se asigna el gasto entre grupos de bienes y, segundo, se asigna el gasto dentro de cada grupo. Al aplicar el método de Rothbarth, se supone que en la primera etapa los padres deciden cómo asignar el presupuesto entre su propio consumo $\left(X^{a}\right)$ y el de sus hijos $\left(X^{c}\right)$. En la segunda etapa, los padres deciden cómo asignar los recursos destinados a cada grupo de bienes entre los diferentes bienes que integran cada grupo. Se considera que no hay bienes públicos, es decir, bienes consumidos tanto por adultos como por niños. Considerando el supuesto de separabilidad, la utilidad de los padres depende exclusivamente del presupuesto asignado a su propio consumo y es independiente del consumo de los niños. Para conocer el gasto necesario para que un hogar con niños alcance un determinado nivel de bienestar, hay que modelar la decisión de asignación de la primera fase (Gronau, 1988).

\section{DATOS}

Los datos empleados corresponden a la base de datos de la Encuesta Nacional de Gastos de los Hogares (ENGHo) de Argentina, realizada a nivel nacional entre marzo de 2012 y marzo de 2013. Esta encuesta incluye aproximadamente 37.000 viviendas seleccionadas, por muestreo probabilístico, polietápico y estratificado, a partir de la Muestra Maestra Urbana de Viviendas de la República Argentina.

La encuesta releva información a nivel desagregado sobre el ingreso de los hogares y el gasto, así como variables sociodemográficas, ocupacionales y educacionales de los miembros de los hogares, características de la vivienda, equipamiento del hogar $\mathrm{y}$ transferencias en especie recibidas.

A los efectos de esta investigación, se seleccionaron hogares de todo el país ${ }^{5}$ compuestos por uno o dos adultos (menores de 65 años), con y sin hijos. En los hogares con niños, se consideraron únicamente aquellos con hasta tres hijos como máximo y

5 La amplitud geográfica reduce el sesgo de localización en las estimaciones (Tsakloglou, 1991). 
de edades que no superen los 14 años ${ }^{6}$. De este modo, la muestra queda conformada por 8.857 observaciones, de estas, el $63,2 \%$ incluyen dos adultos, mientras que el $36,8 \%$ son hogares de un adulto.

La selección de los bienes de adulto, que es la principal fuente de información para nuestra estimación no es un tema trivial. Así como se explicó al analizar las condiciones requeridas para sostener el enfoque de Rothbarth, las estimaciones son sensibles al tipo de bienes incluidos para el cómputo de los gastos atribuibles a los miembros adultos de las familias y es necesario que la demanda de esos bienes sea elástica a los cambios en el presupuesto. Las encuestas de gastos no permiten identificar claramente quién entre los miembros del hogar consume qué bien de los adquiridos por la familia ${ }^{7}$. Debido a ello, lo habitual es incluir aquellos bienes que, a priori, puede sostenerse que los niños están excluidos de su consumo. De este modo, los bienes que se consideran apropiados suelen ser las bebidas alcohólicas, el tabaco, la indumentaria y el calzado de adultos. En la opinión de Schulte (2007), los dos últimos serían los que mejor se adaptan a los requerimientos del modelo fundamentalmente por tres razones: 1) poseen una elasticidad de ingreso alta y cercana a uno, 2) representan una fracción del presupuesto de las familias no despreciable y cercana al $10 \%$ en promedio, y 3 ) probablemente la demanda de estos bienes se vea menos influenciada por la presencia de niños que la de tabaco o alcohol. Deaton (1989) concluyó que el conjunto de bienes de adultos se compone de alcohol, ropa de adultos, tabaco, gastos en salud de adultos, cuidado personal, transporte y educación de adultos. A su vez Tsakloglou (1991), al realizar un test para verificar que el efecto que provoca un niño en el gasto de estos bienes sea significativo, agrega a este listado a las comidas fuera del hogar y el entretenimiento de adultos.

Con el fin de este trabajo y luego de analizar el detalle de los bienes reportados por la Encuesta de Gastos 2012/13 en los distintos rubros, se han considerado para conformar la variable gasto en bienes de adultos a las siguientes categorías: bebidas alcohólicas, consumidas dentro y fuera del hogar (incluidas en el rubro alimentos y bebidas), ropa interior y exterior para hombres y mujeres, artículos de marroquinería y calzado para hombres y mujeres (incluidas en el rubro indumentaria), juegos de azar y diarios y periódicos (incluidos en el rubro esparcimiento) y tabaco, artículos de tocador y servicios para el cuidado personal y cosméticos (incluidos en el rubro bienes

6 La restricción de la muestra al tipo de hogares descriptos es común en la literatura que aplica Rothbarth, debido a que se procura que la composición de los miembros adultos sea lo más similar posible entre el hogar con niños y los hogares de referencia (sin niños). A su vez, la restricción del número de niños en el hogar obedece a que frecuentemente las familias más numerosas presentan valores de escalas negativas para el cuarto o quinto niño (debido a que los hogares relativamente más pobres en la distribución de ingresos totales son también los que presentan un número mayor de niños por hogar). Finalmente el corte de edad para los niños a los 14 años, se relaciona con la estructura de la base de datos que identifica la cantidad de menores de 14 años y el número total de hijos.

7 Aunque se incluyen cartillas para registrar consumos individuales, frecuentemente están incompletas y además la mayor parte de los bienes son adquiridos por el miembro a cargo de esta actividad en el hogar y se describen sin discriminar destinatario, en las categorías correspondientes. 
y servicios varios). Como es esperable, el rubro de indumentaria es el componente con mayor peso relativo en los bienes de adulto.

Un análisis no condicionado muestra que, en promedio, los hogares con niños gastan relativamente menos en bienes de adulto (Tabla 1) y que, al mismo tiempo, estos bienes tienen una menor participación en el gasto total (Tabla 2), en comparación con los hogares sin niños. Se observa que la participación promedio del gasto en bienes de adulto en el presupuesto del hogar es menor en los hogares con niños. Este comportamiento es consistente con lo enunciado por Rothbarth (1943).

\section{TABLA 1}

\section{GASTO PROMEDIO EN BIENES DE ADULTO}

\begin{tabular}{|c|c|c|c|c|c|c|}
\hline \multirow[b]{2}{*}{$\begin{array}{l}\text { Rubro } \\
\text { de Gasto }\end{array}$} & \multicolumn{3}{|c|}{ Hogares con dos adultos } & \multicolumn{3}{|c|}{ Hogares con un adulto } \\
\hline & $\begin{array}{l}\text { Sin } \\
\text { niños }\end{array}$ & $\begin{array}{c}\text { Con } \\
\text { Niños }\end{array}$ & Var. $\%$ & $\begin{array}{l}\text { Sin } \\
\text { niños }\end{array}$ & $\begin{array}{l}\text { Con } \\
\text { niños }\end{array}$ & Var. \% \\
\hline Alimentos y bebidas & $\$ 206,11$ & $\$ 157,99$ & $-23,35$ & $\$ 222,11$ & $\$ 120,08$ & $-45,94$ \\
\hline Indumentaria & $\$ 574,11$ & $\$ 572,53$ & 28 & $\$ 530,42$ & $\$ 417,92$ & $-21,21$ \\
\hline Esparcimiento & $\$ 92,82$ & $\$ 67,85$ & $-26,90$ & $\$ 54,06$ & $\$ 67,15$ & 24,21 \\
\hline Bs. y ss. varios & $\$ 185,53$ & $\$ 158,88$ & $-14,36$ & $\$ 169,60$ & $\$ 149,03$ & $-12,13$ \\
\hline Total & $\$ 674,56$ & $\$ 611,48$ & $-9,35$ & $\$ 582,60$ & $\$ 466,21$ & $-19,98$ \\
\hline
\end{tabular}

Fuente: Elaboración propia a base de datos de la ENGHo 2012/2013.

Nota: Gasto promedio de los hogares que efectivamente gastan en bienes de adulto de cada rubro, de acuerdo con la presencia o no de niños en el hogar, a precios constantes a marzo del 2013.

\section{TABLA 2}

PARTICIPACION PROMEDIO DE LOS BIENES DE ADULTO EN EL PRESUPUESTO DEL HOGAR

\begin{tabular}{|l|c|c|}
\hline & Hogar de un adulto & Hogar de dos adultos \\
\hline Hogar sin niños & $11,76 \%$ & $10,25 \%$ \\
Hogar con niños & $6,18 \%$ & $7,23 \%$ \\
Promedio & $10,30 \%$ & $8,21 \%$ \\
\hline
\end{tabular}

Fuente: Elaboración propia a base de datos de la ENGHo 2012/2013.

Nota: Participación promedio del gasto en bienes de adulto en el presupuesto del hogar, de acuerdo con la presencia de niños en el hogar, a precios constantes a marzo del 2013.

Si bien en promedio los hogares con dos adultos tienen un mayor gasto en bienes de adultos, también poseen una menor participación promedio del gasto en bienes de adultos en el presupuesto del hogar debido a que el nivel de ingreso promedio de los 
hogares con dos adultos es mayor que el de los hogares con un adulto. De esta manera, suponiendo que enfrentan los mismos precios, el impacto en el presupuesto del hogar que genera un niño es mayor, en promedio, para el caso de hogares con un adulto.

\section{ESTRATEGIA EMPIRICA}

La estrategia empírica está basada en la formulación de Schulte (2007). El presupuesto total del hogar $(X)$ está compuesto por el gasto de los adultos $\left(X^{a}\right)$ y el presupuesto de los niños $\left(X^{c}\right)$. A su vez, una porción del presupuesto de los adultos está destinado a los denominados bienes de adultos, esto es, bienes que son consumidos exclusivamente por ellos $\left(X^{b a}\right)$. Dicho gasto en bienes adultos está representado por la siguiente ecuación (2).

$$
X^{b a}=\beta_{0}+\beta_{1} X^{a}
$$

Por su parte, el presupuesto de los niños en el hogar viene dado por (3).

$$
X^{c}=\gamma X k 1, \quad \text { con } k 1=\ln (k+1)
$$

Donde $\gamma$ es el costo relativo de un hijo, $k$ es la cantidad de niños presentes en el hogar, y $k l$ es el logaritmo natural de la cantidad de niños presentes en el hogar más uno.

Por tanto, la ecuación de interés (2) se transforma en la ecuación (4).

$$
X^{b a}=\beta_{0}+\beta_{1}[[1-\gamma \ln (k+1)] X]
$$

De esta manera, al comparar un hogar $s$ con $k^{s}$ niños, con el hogar de referencia $r$ de $k^{r}$ niños, y con el mismo nivel de gasto en bienes de adultos, la escala se estima como indica la expresión (5).

$$
m_{r}^{s}=\frac{X^{s}}{X^{r}}=\frac{1-\gamma \ln \left(k^{r}+1\right)}{1-\gamma \ln \left(k^{s}+1\right)}
$$

Cuando el hogar de referencia $r$ no tiene niños, $k^{r}=0$ y $\ln \left(k^{r}+1\right)=0$. Además, el gasto total del hogar $r$ equivale al gasto en bienes de adultos del hogar $s$, por lo que $X^{r}=X^{a}$. La escala de equivalencia se estima, entonces, de acuerdo a (6).

$$
m^{s}=\frac{X^{s}}{X^{r}}=\frac{1}{1-\gamma \ln \left(k^{s}+1\right)}
$$


A los efectos de reducir la heterocedasticidad, se utiliza como variable dependiente la participación de los bienes de adultos en el presupuesto del hogar, $W_{b a}$, tal como expresa (7).

$$
w_{b a}=\frac{X^{b a}}{X}
$$

De esta manera, la ecuación (4) se transforma de la forma indicada en (8).

$$
w_{b a}=\theta_{0} \frac{1}{X}+\theta_{1}+\theta_{2} \quad k 1
$$

La escala de equivalencia del hogar $s$ con $k^{s}$ hijos, respecto de un hogar de referencia en el que no hay niños, es como se indica en (9).

$$
\widehat{m^{s}}=\frac{1}{1+\frac{\theta_{2}}{\theta_{1}} \ln \left(k^{s}+1\right)}
$$

\subsection{Corrección por sesgo}

Existe una proporción no trivial de hogares que no gasta en bienes de adultos $(17,5 \%)$. Este es un problema bastante frecuente cuando se trabaja con encuestas de gastos en las que las familias reportan los bienes adquiridos en un cierto período de referencia. Sin embargo, el que no hayan efectivamente adquirido ropa en los últimos meses, no implica que no gasten en esos bienes. Si se estimara el costo de los niños y las escalas de equivalencia con los datos de los hogares que efectivamente gastan en bienes de adultos, ignorando a aquellos que no lo hacen, podrían obtenerse estimadores sesgados, debido a que el monto que gasta cada hogar en bienes de adultos puede estar correlacionado con variables que afecten a la decisión de gastar o no en bienes de adultos. En función de ello, y de forma análoga a como se trabaja en las estimaciones de demanda a partir de este tipo de datos, se estiman las escalas de equivalencia aplicando el método de corrección de sesgo de Heckman (1979).

De este modo, se estiman escalas de equivalencia en dos etapas. En la primera, se computa una regresión probabilística que permite estimar la probabilidad de que los hogares gasten en bienes de adultos, para corregir el sesgo de selección. En este caso, la variable dependiente de la ecuación de selección es si el hogar gasta o no gasta en bienes de adultos. Como variables explicativas se incluyen: variables indicadoras de los quintiles de ingreso, de la presencia de niños en el hogar, de la región del país a la que pertenece, del nivel educativo (primaria o secundaria) y género del jefe del hogar, así como la cantidad de miembros ocupados en el hogar. En el caso de los hogares de 
un único adulto, el vector de variables dependientes incluye a las referidas al quintil al que pertenece el hogar, la presencia de niños en el hogar, si el hogar pertenece a la región patagónica, el sexo del jefe del hogar, si el jefe del hogar está ocupado y si usa transporte público ${ }^{8}$. En la segunda etapa, se modela por Mínimos Cuadrados Ordinarios la ecuación de interés (8) aumentada por el inverso del ratio de Mills (estimado en la primera etapa), y considerando únicamente a los hogares que incurren en gastos de bienes de adultos. Entonces, la ecuación final a estimar es la (10).

$$
w_{b a}=\theta_{1}+\theta_{2} \quad k 1+\gamma Z++\theta_{7} \hat{\lambda}_{i}+u
$$

El análisis es realizado considerando por separado los dos tipos de hogares. En (10) el vector $Z$ incluye la inversa del ingreso $(1 / X)$ y las variables explicativas del modelo probit a excepción de las referidas a nivel educativo del jefe del hogar -en el caso de hogares de dos adultos- y el uso de transporte público -en el caso de un adulto- ${ }^{9}$. Asimismo, tampoco se incluyen las variables de quintiles de ingreso, debido al empleo del presupuesto total, y la indicadora de hijos en el hogar, que se sustituye por el número de niños.

\subsection{Escalas por edad y sexo}

Con el efecto de distinguir el impacto en el bienestar que tiene cada niño en el hogar, se computan escalas diferenciando por la edad de los niños. En particular, se considera como corte de edad los 5 años. En este caso, la ecuación a estimar es (11).

$$
w_{b a}=\theta_{1}+\theta_{21} \quad k 1 m a y+\theta_{21} \quad k 1 m e n+\gamma Z++\theta_{7} \widehat{\lambda}_{i}+u
$$

Donde $k 1$ may se refiere a la cantidad de niños mayores a 5 años en el hogar, y $k 1 m e n$ a la cantidad de niños menores a 5 años presentes en el hogar.

De este modo, la escala de equivalencia para un hogar $s$ con $k^{\text {may }}$ niños mayores a 5 años de edad y $k^{m e n}$ niños de 5 años de edad o menos, respecto de un hogar de referencia en el que no hay niños, se transforma en (12).

8 Se han considerado distintas variables para la estimación de la primera etapa para hogares de dos adultos o de un adulto en pro de elegir aquel modelo que mejor se ajuste a los datos en cada uno de los casos. En particular, para el segundo tipo de hogar, no resultaron significativas las variables referidas al nivel educativo del jefe del hogar y se agregó la variable indicadora del uso de transporte público. La inclusión de esta última resulta significativa y de signo positivo y una explicación posible es que, para estos hogares de menor ingreso relativo, la probabilidad de gastar en bienes de adultos es mayor si el único adulto debe desplazarse, saliendo fuera del hogar para trabajar, respecto de permanecer en él.

9 Esta es una exigencia del método de Heckman tal que el conjunto de variables explicativas en la segunda etapa debe ser un subconjunto de las intervinientes en la primera etapa. 


$$
\widehat{m^{s}}=\frac{1}{1+\frac{\theta_{21}}{\theta_{1}} \ln \left(k^{m a y}+1\right)+\frac{\theta_{22}}{\theta_{1}} \ln \left(k^{m e n}+1\right)}
$$

Finalmente, para analizar el impacto que tiene cada niño en el hogar, distinguiendo por su género, la ecuación de demanda de bienes de adultos es (13).

$$
w_{b a}=\theta_{1}+\theta_{21} \quad k 1 \text { mujeres }+\theta_{21} \quad k 1 \text { varones }+\gamma Z++\theta_{7} \quad \hat{\lambda}_{i}+u
$$

Mientras que la escala de equivalencia para un hogar $s$ con $k^{\text {mujeres }}$ niñas y $k^{\text {varones }}$ niños, respecto de un hogar de referencia en el que no hay niños, se estima de forma análoga a la ecuación 12 .

\section{RESULTADOS}

En esta sección se presentan los resultados de las escalas de equivalencia estimadas para los dos tipos de hogares considerados (hogares de dos padres y monoparentales). Asimismo, se presentan las escalas diferenciadas por edad y género de los niños. En el Anexo se reportan las regresiones correspondientes a la primera (modelo probabilístico) y segunda (modelo lineal) etapa de cada estimación. En todas ellas, el coeficiente de interés estimado en la primera etapa (inverso del ratio de Mills) es significativo, denotando la necesidad de corregir por el problema de selección presente. Por último, se comparan las escalas obtenidas en esta investigación con aquellas obtenidas por Pace Guerrero (2013) bajo otro método de identificación y con los resultados de otros trabajos empíricos que estiman escalas de Rothbarth.

\subsection{Escalas por tipo de hogar}

En la Tabla 3 se reportan las escalas de equivalencia para los hogares biparentales, mientras que en la Tabla 4 las escalas para los hogares monoparentales. La incorporación al hogar de un niño en hogares de dos adultos se relaciona a un incremento del gasto total del hogar de $31 \%$, y del $41 \%$ en hogares de un adulto. Los hogares con dos o tres hijos requieren de aumentos en el gasto proporcionalmente menores para alcanzar el mismo nivel de bienestar que si no tuvieran hijos. Al mismo tiempo, se evidencia que los valores de las escalas para los hogares monoparentales son más elevadas. 
TABLA 3

ESCALAS DE EQUIVALENCIA ESTIMADAS PARA HOGARES DE DOS ADULTOS

\begin{tabular}{|c|c|c|c|}
\hline & $\begin{array}{l}\text { Dos adultos } \\
\text { y un niño }\end{array}$ & $\begin{array}{l}\text { Dos adultos } \\
\text { y dos niños }\end{array}$ & $\begin{array}{l}\text { Dos adultos } \\
\text { y tres niños }\end{array}$ \\
\hline & $\begin{array}{c}1,31^{* * * *} \\
(0,39)\end{array}$ & $\begin{array}{c}1,60^{* * *} \\
(0,92)\end{array}$ & $\begin{array}{c}1,89^{* * * *} \\
(0,16)\end{array}$ \\
\hline Incremento porcentual de la escala & $31 \%$ & $22,1 \%$ & $18,6 \%$ \\
\hline $\mathrm{N}$ & 1.717 & 1.666 & 655 \\
\hline
\end{tabular}

Fuente: Elaboración propia a base de datos de la ENGHo 2012/2013.

Nota: El hogar de referencia de las escalas es el constituido por dos adultos de hasta 65 años sin niños. $* * * \mathrm{p}<0,01, * * \mathrm{p}<0,05, \mathrm{y} * \mathrm{p}<0,1$. Errores estándar estimados por el método delta entre paréntesis.

\section{TABLA 4}

ESCALAS DE EQUIVALENCIA ESTIMADAS PARA HOGARES DE UN ADULTO

\begin{tabular}{|l|c|c|c|}
\hline & Un adulto & Un adulto & Un adulto \\
& y un niño & dos niños & y tres niños \\
\hline Escala de equivalencia & $1,41^{* * *}$ & $1,86^{* * *}$ & $2,40^{* * *}$ \\
& $(0,09)$ & $(0,25)$ & $(0,54)$ \\
Incremento porcentual de la escala & $41,3 \%$ & $31,8 \%$ & $29,2 \%$ \\
\hline $\mathrm{N}$ & 403 & 253 & 96 \\
\hline
\end{tabular}

Fuente: Elaboración propia a base de datos de la ENGHo 2012/2013.

Nota: El hogar de referencia de las escalas es el constituido por un adulto de hasta 65 años sin niños. $* * * \mathrm{p}<0,01, * * \mathrm{p}<0,05, \mathrm{y} * \mathrm{p}<0,1$. Errores estándar estimados por el método delta entre paréntesis.

\subsection{Escalas por edad y sexo}

El costo de los niños puede ser distinto según sus características. En la Tabla 5 se presentan las escalas estimadas para los hogares de dos adultos y un adulto con distinto número de niños, considerando los 5 años como corte relevante de edad, mientras que en el Tabla 6 se presentan las escalas considerando la composición de género de los niños del hogar.

Los resultados sugieren que los gastos destinados a bienes de adultos experimentan una mayor reducción si los niños pertenecen al tramo superior de edad, mientras que las mujeres suponen una reducción mayor que los varones en idéntico tramo de edad.

De la comparación de las estimaciones surge una observación, relacionada con el costo de los niños, que describe un comportamiento de gastos en los hogares bastante plausible. De acuerdo con las escalas estimadas, una pareja de adultos que tuviera 


\section{TABLA 5}

ESCALAS DE EQUIVALENCIA ESTIMADAS SEGUN EL GRUPO ETARIO

\begin{tabular}{|c|c|c|c|}
\hline \multirow{2}{*}{$\begin{array}{c}\text { Cantidad de niños }> \\
5 \text { años }\end{array}$} & Cantidad de niños $\leq$ & \multicolumn{2}{|c|}{ Escala de equivalencia } \\
\cline { 2 - 4 } & 5 años & Hogares de dos adultos & Hogares de un adulto \\
\hline 1 & 0 & $1,26^{* * * *}(0,41)$ & $1,39^{* * * *}(0,10)$ \\
0 & 1 & $1,23^{* * * *}(0,04)$ & $1,45^{* * * *}(0,13)$ \\
1 & 1 & $1,66^{* * *}(0,11)$ & $2,46^{* * * *}(0,55)$ \\
2 & 0 & $1,49^{* * * *}(0,09)$ & $1,81^{* * *}(0,28)$ \\
0 & 2 & $1,43^{* * *}(0,08)$ & $1,97^{* * *}(0,40)$ \\
2 & 1 & $2,09^{* * * *}(0,23)$ & $4,14^{* * *}(1,98)$ \\
1 & 2 & $2,05^{* * *}(0,22)$ & $4,45^{*}(2,46)$ \\
3 & 0 & $1,71^{* * * *}(0,15)$ & $2,30^{* * *}(0,57)$ \\
0 & 3 & $1,62^{* * *}(0,13)$ & $2,64^{* * *}(0,92)$ \\
\hline
\end{tabular}

Fuente: Elaboración propia a base de datos de la ENGHo 2012/2013.

Nota: El hogar de referencia de las escalas es el constituido por dos o un adulto, respectivamente, de hasta 65 años sin niños. *** $\mathrm{p}<0,01, * * \mathrm{p}<0,05, \mathrm{y} * \mathrm{p}<0,1$. Errores estándar estimados por el método delta entre paréntesis.

\section{TABLA 6}

ESCALAS DE EQUIVALENCIA ESTIMADAS SEGUN EL SEXO

\begin{tabular}{|c|c|c|c|}
\hline \multirow{2}{*}{ Cantidad de niñas } & \multirow{2}{*}{ Cantidad de niños } & \multicolumn{2}{|c|}{ Escala de equivalencia } \\
\cline { 3 - 4 } & & Hogares de dos adultos & Hogares de un adulto \\
\hline 1 & 0 & $1,25^{* * * *}(0,04)$ & $1,35^{* * * *}(0,10)$ \\
0 & 1 & $1,25^{* * * *}(0,04)$ & $1,42^{* * * *}(0,12)$ \\
1 & 1 & $1,67^{* * * *}(0,11)$ & $2,27^{* * *}(0,44)$ \\
2 & 0 & $1,47^{* * * *}(0,09)$ & $1,71^{* * * *}(0,27)$ \\
0 & 2 & $1,46^{* * *}(0,08)$ & $1,90^{* * *}(0,34)$ \\
2 & 1 & $2,09^{* * * *}(0,23)$ & $3,50^{* * *}(1,40)$ \\
1 & 2 & $2,09^{* * * *}(0,22)$ & $3,78^{* *}(1,64)$ \\
3 & 0 & $1,68^{* * *}(0,14)$ & $2,10^{* * * *}(0,52)$ \\
0 & 3 & $1,67^{* * * *}(0,14)$ & $2,48^{* * *}(0,73)$ \\
\hline
\end{tabular}

Fuente: Elaboración propia a base de datos de la ENGHo 2012/2013.

Nota: El hogar de referencia de las escalas es el constituido por dos o un adulto, respectivamente, de hasta 65 años sin niños. *** $\mathrm{p}<0,01, * * \mathrm{p}<0,05, \mathrm{y} * \mathrm{p}<0,1$. Errores estándar estimados por el método delta entre paréntesis.

3 niños, gastaría $89,91 \%$ más que si no los tuvieran, pero si los 3 fueran menores de 5 años, los requerimientos descenderían al 62,62\% de gastos adicionales. Por el contrario, la mezcla de edades disminuiría la posibilidad de economías de escala en 
los gastos de forma que, 2 niños en primaria (mayores de 5 años) y uno en preescolar, implicaría un costo equivalente a 109,57\% adicional al correspondiente a los dos adultos. De la misma forma, la diferencia de sexo de los niños también atenta contra las economías de escala, mientras que 3 niñas implican un costo adicional para una pareja de $68,64 \%$, dos niñas y un varón aumentan ese costo a 109,76\%.

Se observan diferencias en el costo de los niños según el rango etario de los niños. En los hogares monoparentales los gastos destinados a bienes de adultos experimentan una mayor reducción si los niños pertenecen al tramo inferior de edad. Asimismo, los niños varones suponen una reducción mayor que las mujeres en idéntico tramo de edad.

\subsection{Comparación de escalas}

En la Tabla 7 se comparan las escalas asociadas al costo de los niños con las obtenidas por Pace Guerrero (2013) con encuestas de gasto pero bajo otro enfoque de identificación ${ }^{10}$. Si bien no es posible realizar una comparación exacta entre los métodos, porque se utilizaron encuestas correspondientes a distintos años y se estimaron escalas considerando distintos cortes de edad, se observa que las escalas obtenidas en este trabajo resultan inferiores. Esto resulta evidencia a favor de la hipótesis de Tsakloglou (1991), según este, el método de Rothbarth subestima las escalas de equivalencia.

\section{TABLA 7}

COMPARACION DE ESCALAS ESTIMADAS EN ARGENTINA

\begin{tabular}{|l|c|c|c|c|c|}
\hline & $\begin{array}{c}\text { Barten } \\
96-97\end{array}$ & \multicolumn{2}{|c|}{$\begin{array}{c}\text { Barten } \\
04-05\end{array}$} & \multicolumn{3}{|c|}{$\begin{array}{c}\text { Rothbarth } \\
12-13\end{array}$} \\
\cline { 2 - 6 } & \multicolumn{2}{|c|}{1 niño } & 1 niño & 2 niños & 3 niños \\
\hline 0 a 2 años & 1,01 & 1,04 & 0,23 & 0,42 & 0,61 \\
3 a 5 años & 0,95 & 1,18 & & 0,48 & 0,70 \\
6 a 9 años \\
10 a 14 años
\end{tabular}

Fuente: Elaboración propia.

Nota: Las escalas Barten son las estimadas por Pace Guerrero (2013) con datos de la ENGHo 1996/97 y 2004/05, respectivamente. Las escalas de Rothbarth se corresponden con las estimaciones obtenidas para el hogar de dos adultos con la ENGHo 2012/13.

10 Otros trabajos empíricos han obtenido escalas de equivalencia para Argentina. Sin embargo, dichas estimaciones han sido realizadas para distintos tipos de hogares representativos, de modo que no es factible diferenciar en ellas el costo relativo de los niños del costo relativo de los adultos. Esto dificulta la comparación con las escalas de Rothbarth, en tanto solo permiten capturar el costo de los niños. 
A los efectos de comparar los resultados de las escalas de Rothbarth estimadas en este trabajo con las reportadas en otros trabajos que utilizaron el mismo modelo, se incluye la Tabla 8 .

\section{TABLA 8} COMPARACION DE ESCALAS ESTIMADAS PARA VARIOS PAISES.
MODELO DE ROTHBARTH

\begin{tabular}{|l|c|c|c|c|c|}
\hline \multirow{2}{*}{ Referencias } & \multirow{2}{*}{$\begin{array}{c}\text { Año de los } \\
\text { datos }\end{array}$} & País & \multicolumn{3}{c|}{ Escalas } \\
\cline { 4 - 6 } & $2012 / 13$ & Argentina & 1,31 & 1,60 & 1,89 \\
\hline $\begin{array}{l}\text { Este trabajo } \\
\text { (hogares biparentales) }\end{array}$ & 1993 & Alemania & 1,21 & 1,38 & 1,53 \\
Schulte (2007) (1) & & Occidental & & & \\
Lancaster y Ray (1998) (2) & $1984 / 88-89$ & Australia & 1,15 & 1,32 & 1,52 \\
Alonzo y Mancero (2011) (3) & & & & & \\
& 1996 & Argentina & 2,38 & & 2,49 \\
& 2003 & Bolivia & 2,51 & & 2,92 \\
& 2002 & Brasil & 2,46 & & 2,75 \\
& 2007 & Chile & 2,49 & & 2,85 \\
& 2007 & Colombia & 2,40 & & 2,57 \\
& 2003 & Ecuador & 2,57 & & 3,13 \\
& 2006 & Guatemala & 2,53 & & 2,98 \\
& 2004 & Honduras & 2,63 & & 3,38 \\
& 2006 & México & 2,61 & & 3,31 \\
& 2005 & Nicaragua & 2,52 & & 2,95 \\
& 2007 & Panamá & 2,51 & & 2,91 \\
& 2003 & Perú & 2,59 & & 3,23 \\
& 1998 & R. Dominicana & 2,50 & & 2,90 \\
& 2006 & Uruguay & 2,28 & & 2,19 \\
\hline
\end{tabular}

Notas: (1) La formulación del modelo es la utilizada en el presente trabajo y los bienes de adulto incluyen indumentaria de hombres y mujeres, bebidas alcohólicas y joyas. (2) La estimación se realizó con otra forma funcional y considerando como bienes de adultos solo ropa. Los valores son más altos considerando como bien de adulto, los gastos de educación de los adultos. (3) Estos autores estiman una forma funcional para obtener el número de adultos equivalentes y los miembros adultos valen 1 , a su vez reportan los casos de hogares con 1,3 y 5 niños. El trabajo considera ropa y calzado como bienes de adulto. Considera niños a los menores de 18 años. Se incluyeron solo los países en donde los parámetros fueron significativos y se informa el modelo de mejor comportamiento.

Es importante mencionar que los resultados no son estrictamente comparables debido a que las estimaciones no son homogéneas en cuanto a su forma funcional y al tipo de bienes de adulto considerado. Asimismo, ninguno de los trabajos reportados tiene en cuenta la edad y el género de los niños. No obstante ello, el costo de los niños es menor en los países de mayor desarrollo como Alemania y Australia, en 
concordancia con las observaciones de Deaton (1997) acerca del comportamiento más inelástico de la demanda de bienes de adulto en estos países.

Respecto de los países latinoamericanos, las estimaciones de Alonzo y Mancero (2011) indican un costo marginal para el primer niño dentro de un rango 28\%-73\% y para el tercero la mayoría de los valores se corresponden con un costo adicional de 10 a $25 \%$ del presupuesto del hogar. En términos del presente trabajo, los mismos valores resultan $31 \%$ y $18,6 \%$, respectivamente.

\section{CONCLUSIONES}

El objetivo de este trabajo consiste en estimar el costo derivado de la incorporación de niños al hogar para Argentina bajo el método propuesto por Rothbarth (1943), focalizando en hogares de dos padres y monoparentales.

Las escalas de equivalencia estimadas son más altas a medida que se incorporan más niños al hogar. Sin embargo, se observa evidencia de economías de escala en el consumo de la familia; a medida que aumenta la cantidad de niños en el hogar, las escalas se incrementan en una proporción cada vez menor.

Asimismo, el costo de los niños es mayor para los hogares monoparentales. En principio, esto se asocia al menor nivel de ingreso promedio de hogares conformados por un único adulto. Suponiendo que ambos tipos de hogares enfrentan a los mismos precios, los bienes y servicios destinados a los niños representan una participación en el presupuesto del hogar mayor para hogares conformados por un adulto.

Los resultados evidencian que las escalas de equivalencia correspondientes a una misma cantidad de niños en el hogar difieren en función del sexo y la edad de los niños. Si los niños en un mismo hogar pertenecen a rangos etarios diferentes -según sean menores o mayores de cinco años- o son de distinto sexo, los costos que enfrenta el hogar para mantener el bienestar de referencia serán más elevados. Estas diferencias se relacionan posiblemente con la presencia de economías de escala en el consumo de las familias. El hecho que los niños tengan edades similares o sean del mismo sexo, implica la posibilidad de compartir juegos, útiles y usar ropa de sus hermanos. Siempre más hijos estarán asociados a mayores gastos aunque no aumentarán en la misma proporción, el primero será más costoso que el segundo y este más que el tercero. Esa proporción será tanto menor cuanto más similares sean las necesidades y requerimientos de los niños.

Asimismo, mientras que los niños de mayor edad son los proporcionalmente más caros para el hogar conformado por una pareja, los de menor edad son los relativamente más costosos para el hogar con un solo adulto a cargo. Parece razonable que en los hogares de dos padres los niños de mayor edad tengan mayores requerimientos específicos asociados, por ejemplo, a su educación en comparación con los niños pequeños. Este efecto puede ser más fuerte si consideramos que muchos de los niños de familias biparentales, que poseen un ingreso relativamente más alto que las monoparentales, 
asisten a colegios privados. Por su parte, en hogares monoparentales, caracterizados por tener menor ingreso en promedio, resulta razonable que un niño pequeño implique gastos más altos (especialmente en pañales o en ciertos requerimientos esenciales que requieren mayor presupuesto), en tanto los niños de mayor edad posiblemente reciban parte de su alimentación en el colegio o subsidios para la compra de útiles escolares en escuelas públicas.

Por último, se evidencia que las niñas son relativamente más caras para el hogar de una pareja mientras que los niños lo son para el hogar monoparental. Una posible explicación puede relacionarse con la "discriminación" en términos de gasto (Deaton, 1989), es decir, las preferencias redistributivas de los padres al interior del hogar de acuerdo con el género de los hijos. En este caso, debido a que la mayoría de los hogares monoparetales están conformados por jefas mujeres, las madres podrían tener una mayor preferencia en el gasto hacia los niños, reasignando más de su propio consumo. Por su parte, en los hogares de dos padres las preferencias de los adultos podrían estar más balanceadas o contrarrestarse, dependiendo del proceso de negociación entre la pareja. Sin embago, no es posible afirmar esto sin adoptar un enfoque que modele la distribución de recursos intrahogar, considerando las preferencias individuales y el poder de negociación de cada integrante de la familia.

Finalmente, a partir de estos resultados se pueden proponer algunas recomendaciones que podrían ser de utilidad para el diseño de políticas focalizadas en las familias con niños. En primer lugar, que cualquier programa de ayuda que se implemente con destino a hogares vulnerables debería considerar especialmente a los hogares a cargo de un único miembro adulto. En segundo lugar, que el número de niños en el hogar, teniendo en cuenta edades y género diferentes, implican requerimientos también diferentes que tendrían menos posibilidades de compartirse, aumentando los gastos necesarios para garantizar un mínimo de bienestar aceptable para todos los hogares.

También es importante agregar que las escalas de Rothbarth, así como las estimadas en esta investigación, en concordancia con la hipótesis que sostiene que subestiman "el verdadero valor" de equivalencia en términos de bienestar, resultan efectivamente menores que las escalas calculadas por expertos. En ese sentido, las escalas de Rothbarth podrían suministrar mínimos valores entre un rango posible de equivalencias a considerar, de forma que cualquier compensación monetaria que se acordara debería al menos ser mayor que el piso asignado por estos valores.

\section{REFERENCIAS}

ALONZO, H. y X. MANCERO (2011). "Escalas de equivalencia en los países de América Latina". CEPAL. Chile.

BARGAIN, O. y O. DONNI (2012). "Expenditure on children: A Rothbarth-type method consistent with scale economies and parents' bargaining". European Economic Review, 56(4), pp. 792-813.

BARGAIN, O.; O. DONNI y P. KWENDA (2014). "Intrahousehold distribution and poverty: Evidence from Cote d'Ivoire". Journal of Development Economics, 107, pp. 262-276. 
BERGES, M. (2010). "Estimación de escalas de equivalencia para Argentina". Tesis de Doctorado, Universidad Nacional de La Plata. Argentina.

BERGES, M. (2011). "Escalas de equivalencia y cambios en el nivel de bienestar de los hogares de la ciudad de Buenos Aires". Revista de Economía Política de Buenos Aires, 5(9-10), pp. 41-90.

BETSON, D. M. y J. MUELLBAUER (2004). "Poverty equivalence scales: adjustment for demographic differences across families". In National Research Council Workshop on Experimental Poverty Measures. Estados Unidos.

BLACKORBY, C. y D. DONALDSON (1988). "Money metric utility: A harmless normalization?" Journal of Economic Theory, 46(1), pp. 120-129.

BROWNING, M.; P.A. CHIAPPORI y A. LEWBEL (2013). "Estimating consumption economies of scale, adult equivalence scales, and household bargaining power". Review of Economic Studies, 80(4), pp. 1267-1303.

CHIAPPORI, P. A. (1988). "Rational household labor supply". Econometrica: Journal of the Econometric Society, pp. 63-90.

CHIAPPORI, P. A. (1992). "Collective labor supply and welfare”. Journal of political Economy, 100(3), pp. 437-467.

CHIAPPORI, P. A. (2016). "Equivalence versus indifference scales". The Economic Journal, 126 (592), pp. 523-545.

COULTER, F.; F. COWELL y S. JENKINS (1992). "Equivalence scales relativities and the extent of inequality and poverty". The Economic Journal, Vol. 102(414), pp. 1067-1082.

DE VOS, K. y M.A. ZAIDI (1997). "Equivalence Scale Sensitivity of Poverty Statisticsfor the Member States of the European Community". Review of Income and Wealth, Vol. 43(3), pp. 319-334.

DEATON, A. (1997). "The analysis of household surveys: a microeconometric approach to development policy". The World Bank. Estados Unidos.

DEATON, A. S.; J. RUIZ-CASTILLO y D. THOMAS. (1989). "The influence of household composition on household expenditure patterns: theory and Spanish evidence". Journal of Political Economy, 97(1), pp. $179-200$.

DEATON, A. y J. MUELLBAUER (1980). "Economics and Consumer Behavior”. Cambridge University Press. 2da Edición

ECHEVERRIA, L. y M. BERGES (2015). "Equivalencias entre gastos de subsistencia para hogares tipo de la Ciudad Autónoma de Buenos Aires". FACES, 21(44), pp. 27-48.

ECHEVERRIA, L.; M. MENON; F. PERALI y M. BERGES (2019). "Intra-Household Inequality and Child Welfare in Argentina". Centro de Estudios Distributivos, Laborales y Sociales. CEDLAS Working Paper Series, $\mathrm{N}^{\circ} 241$

GRONAU, R. (1988). "Consumption technology and the intrafamily distribution of resources: adult equivalence scales reexamined". Journal of Political Economy, 96(6), pp. 1183-1205.

GROOTAERT, C. (1982). "The conceptual basis of measures of household welfare and their implied survey data requirements". Development Research Dept., $\mathrm{N}^{\circ} 19$, World Bank. Estados Unidos.

INSTITUTO NACIONAL DE ESTADISTICAS Y CENSOS (2013). "Encuesta Nacional de Gastos de los Hogares 2012/2013”. Resumen metodológico. Argentina.

LANCASTER, G. y R. Ray (1998). "Comparison of alternative models of household equivalence scales: The Australian evidence on unit record data". Economic Record, 74(224), pp. 1-14.

LAZEAR, E. P. y R. T. MICHAEL (1980). "Family size and the distribution of per capita income". American Economic Review, 70, pp. 91-107.

LEWBEL, A. (1989). "Household equivalence scales and welfare comparisons". Journal of Public Economics, 39(3), pp. 377-391.

LEWBEL, A. (2003). "Calculating compensation in cases of wrongful death". Journal of Econometrics, 113(1), pp. $115-128$.

MANCERO, X. (2001). "Escalas de equivalencia: Reseña de conceptos y métodos". Serie Estudios Estadísticos y Prospectivos. 8. Publicación de las Naciones Unidas, División de Estadística y Proyecciones Económicas. Chile.

NELSON, J. A. (1992). "Methods of estimating household equivalence scales: an empirical investigation". Review of Income and Wealth, 38(3), pp. 295-310. 
PACE GUERRERO, I. (2013). "Escalas de Barten, ¿qué nos dicen los datos de consumo acerca de las escalas de equivalencia entre los hogares argentinos?" Anales de la Asociación Argentina de Economía Política. Argentina

ROTHBARTH, E. (1943). "Note on a method of determining equivalent income for families of different composition". In Appendix IV in War-Time Pattern of Saving and Expenditure by Charles Madge, University Press, Cambridge.

SCHULTE, J. N. (2007). "Equivalence scales: identification and estimation". Doctoral dissertation, Freie Universität Berlin. Alemania.

TSAKLOGLOU, P. (1991). "Estimation and comparison of two simple models of equivalence scales for the cost of children”. The Economic Journal, 101(405), pp. 343-357. 


\begin{abstract}
ANEXO
En la Tabla A.1 se presentan los resultados de las estimaciones correspondientes a la primera y segunda etapa de la submuestra de los hogares compuestos por dos adultos, mientras que en la Tabla A.2 se presentan los resultados de ambas etapas correspondientes a los hogares compuestos por un adulto. Asimismo, en las Tablas A.3 y A.4, se reportan para cada tipo de hogar, respectivamente, los resultados correspondientes a la segunda etapa de las estimaciones que diferencian por grupo etario y género de los niños.
\end{abstract}

\title{
TABLA A.1
}

ESTIMACIONES PARA HOGARES CON DOS ADULTOS

\begin{tabular}{|c|c|c|}
\hline Variables & $\begin{array}{l}\text { Primera etapa } \\
\text { Modelo probit }\end{array}$ & $\begin{array}{l}\text { Segunda etapa } \\
\text { Modelo lineal }\end{array}$ \\
\hline $\begin{array}{l}\text { Presencia de niños } \\
\text { Ln (cantidad de niños }+1 \text { ) } \\
1 \text { si pertenece a los quintiles } 1 \text { o } 2 \\
1 \text { si pertenece a los quintiles } 3 \text { o } 4 \\
\text { Inversa del ingreso } \\
1 \text { si pertenece al Gran Bs As } \\
1 \text { si pertenece a la región Patagónica } \\
1 \text { si el jefe tiene educación primaria } \\
1 \text { si el jefe tiene educación secundaria } \\
1 \text { si la jefa del hogar es mujer } \\
\text { Cantidad de ocupados } \\
\text { Lambda (IMR) } \\
\text { Constante }\end{array}$ & $\begin{array}{c}0,126 * * *(0,048) \\
- \\
-1,295 * * *(0,073) \\
-0,541 * * *(0,069) \\
- \\
0,251 * * *(0,077) \\
-0,216 * * *(0,053) \\
0,277 * * *(0,060) \\
0,218 * * *(0,052) \\
0,120 * *(0,055) \\
0,050(0,037) \\
- \\
1,423 * * *(0,092)\end{array}$ & $\begin{array}{c}- \\
-0,032^{* * *}(0,002) \\
- \\
- \\
10,34 *(5,687) \\
0,002(0,004) \\
0,002(0,003) \\
- \\
- \\
0,009^{* * *}(0,003) \\
0,005^{* *}(0,002) \\
0,045^{* * *}(0,009) \\
0,093^{* * *}(0,005)\end{array}$ \\
\hline $\begin{array}{l}\text { AIC } \\
\text { BIC } \\
\mathrm{N}\end{array}$ & & $\begin{array}{c}-4857,9 \\
-4732,44\end{array}$ \\
\hline
\end{tabular}

Fuente: Estimación propia a base de datos de la ENGHo 2012/2013.

Nota: En el modelo probit la variable dependiente toma el valor 1 si el hogar gasta en bienes adultos. En el modelo lineal la variable dependiente es la participación de los bienes de adulto en el gasto total. El modelo lineal es estimado por máxima verosimilitud. *** $\mathrm{p}<0,01$, ** $\mathrm{p}<0,05, \mathrm{y}$ $* \mathrm{p}<0,1$. Errores estándar entre paréntesis. 
TABLA A.2

ESTIMACIONES PARA HOGARES CON UN ADULTO

\begin{tabular}{|c|c|c|}
\hline Variables & $\begin{array}{l}\text { Primera etapa } \\
\text { Modelo probit }\end{array}$ & $\begin{array}{l}\text { Segunda etapa } \\
\text { Modelo lineal }\end{array}$ \\
\hline $\begin{array}{l}\text { Presencia de niños } \\
\text { Ln (cantidad de niños }+1 \text { ) } \\
1 \text { si pertenece a los quintiles } 1 \text { o } 2 \\
1 \text { si pertenece a los quintiles } 3 \text { o } 4 \\
\text { Inversa del ingreso } \\
1 \text { si pertenece a la región Patagónica } \\
1 \text { si la jefa del hogar es mujer } \\
1 \text { si el jefe de hogar está ocupado } \\
1 \text { si el jefe usa transporte público } \\
\text { Lambda (IMR) } \\
\text { Constante }\end{array}$ & $\begin{array}{c}0,124 *(0,074) \\
- \\
-1,264 * * *(0,084) \\
-0,629 * * *(0,070) \\
- \\
-0,252 * * *(0,073) \\
-0,388 * * *(0,067) \\
0,252 * * *(0,068) \\
0,117 *(0,061) \\
- \\
1,414 * * *(0,090)\end{array}$ & $\begin{array}{c}- \\
-0,054 * * *(0,007) \\
- \\
- \\
12,06^{* *}(5,06) \\
-0,017 * * *(0,006) \\
-0,019 * * *(0,005) \\
0,014 * *(0,006) \\
- \\
0,026^{*}(0,016) \\
0,128^{* * *}(0,008)\end{array}$ \\
\hline $\begin{array}{l}\text { AIC } \\
\text { BIC } \\
\mathrm{N}\end{array}$ & & $\begin{array}{l}-807,50 \\
-712,34\end{array}$ \\
\hline
\end{tabular}

Fuente: Estimación propia a base de datos de la ENGHo 2012/2013.

Nota: En el modelo probit la variable dependiente toma el valor 1 si el hogar gasta en bienes adultos. En el modelo lineal la variable dependiente es la participación de los bienes de adulto en el gasto total. El modelo lineal es estimado por máxima verosimilitud. *** $\mathrm{p}<0,01, * * \mathrm{p}<0,05, \mathrm{y}$ $* \mathrm{p}<0,1$. Errores estándar entre paréntesis. 
TABLA A.3

ESTIMACIONES POR GRUPO ETARIO Y SEXO. HOGARES CON DOS ADULTOS (SEGUNDA ETAPA)

\begin{tabular}{|l|c|c|}
\hline \multicolumn{1}{|c|}{ Variables } & $\begin{array}{c}\text { Grupos de edad } \\
\text { Modelo lineal }\end{array}$ & $\begin{array}{c}\text { Género } \\
\text { Modelo lineal }\end{array}$ \\
\hline Cantidad de ocupados & $0,005^{* *}(0,002)$ & $0,004 *(0,0026)$ \\
Ln (cantidad de niños $>5$ años+ 1) & $-0,027^{* * *}(0,003)$ & - \\
Ln (cantidad de niños $\leq 5+1)$ & $-0,025^{* * *}(0,003)$ & - \\
Ln (cantidad de niñas + 1) & - & $-0,027 * * *(0,003)$ \\
Ln (cantidad de niños + 1) & - & $-0,026^{* * *}(0,003)$ \\
Inversa del ingreso & $10,09^{*}(5,69)$ & $10,29 *(5,693)$ \\
1 si pertenece al Gran Bs. As. & $0,003^{* * *}(0,004)$ & $0,002(0,004)$ \\
1 si pertenece a la región Patagónica & $0,002(0,003)$ & $0,002(0,003)$ \\
1 si la jefa del hogar es mujer & $0,010^{* * *}(0,003)$ & $0,010^{* * * *}(0,003)$ \\
Lambda (IMR) & $-0,044^{* * *}(0,009)$ & $0,044 * * *(0,009)$ \\
Constante & $-0,091^{* * *}(0,005)$ & $0,092 *(0,005)$ \\
\hline AIC & $-4847,82$ & $-4850,82$ \\
BIC & $-4713,86$ & $-4716,86$ \\
N & \multicolumn{2}{|c|}{5,996} \\
\hline
\end{tabular}

Fuente: Estimación propia a base de datos de la ENGHo 2012/2013.

Nota: El modelo lineal es estimado por máxima verosimilitud. La variable dependiente es la participación de los bienes de adulto en el gasto total. $* * * \mathrm{p}<0,01, * * \mathrm{p}<0,05, \mathrm{y} * \mathrm{p}<0,1$. Errores estándar entre paréntesis.

TABLA A. 4

ESTIMACIONES POR GRUPO ETARIO Y SEXO. HOGARES CON UN ADULTO (SEGUNDA ETAPA)

\begin{tabular}{|l|c|c|}
\hline \multicolumn{1}{|c|}{ Variables } & $\begin{array}{c}\text { Grupos de edad } \\
\text { Modelo lineal }\end{array}$ & $\begin{array}{c}\text { Género } \\
\text { Modelo lineal }\end{array}$ \\
\hline 1 si el jefe de hogar está ocupado & $0,013 * *(0,006)$ & $0,014 * *(0,006)$ \\
Ln (cantidad de niños $>5$ años+ 1$)$ & $-0,052 * * *(0,008)$ & - \\
Ln (cantidad de niños $\leq 5+1)$ & $-0,057 * * *(0,011)$ & $-0,048 * * *(0,010)$ \\
Ln (cantidad de niñas + 1) & - & $-0,055^{* * *}(0,009)$ \\
Ln (cantidad de niños + 1) & - & $11,97 * *(50,56)$ \\
Inversa del ingreso & $12,09 * *(50,47)$ & $-0,017 * * *(0,006)$ \\
1 si pertenece a la región Patagónica & $-0,017 * * *(0,006)$ & $-0,020 * * *(0,005)$ \\
1 si la jefa del hogar es mujer & $-0,020 * * *(0,005)$ & $0,027 *(0,015)$ \\
Lambda (IMR) & $0,029 *(0,015)$ & $0,128 * * *(0,008)$ \\
Constante & $0,128^{* * * *(0,008)}$ & $-811,67$ \\
\hline AIC & $-813,54$ & $-710,57$ \\
BIC & $-712,44$ & \\
N & & 2,861 \\
\hline
\end{tabular}

Fuente: Estimación propia a base de datos de la ENGHo 2012/2013.

Nota: El modelo lineal es estimado por máxima verosimilitud. La variable dependiente es la participación de los bienes de adulto en el gasto total. $* * * \mathrm{p}<0,01, * * \mathrm{p}<0,05, \mathrm{y} * \mathrm{p}<0,1$. Errores estándar entre paréntesis. 